\title{
Development of a jelly based functional food using natural plant extract of Centella asiatica
}

\author{
By \\ Ramboda Gedara Ajith Kumara
}

Thesis submitted to the University of Sri Jayewardenapura as the partial fulfillment requirement for the award of the degree of Masters of Food Science and Technology

Department of Food Science and Technology, Faculty of Applied science, University of Sri Jayewardenepura, Sri Lanka 


\section{DECLARATION}

I do here by declare that the work reported in this dissertation was exclusively carried out by me under the supervision of Prof. Arthur Bamunuarachchi, Dr Indira Wickramasinghe. It describes the results of my own independent research except where due reference has been made in the text. No part of this dissertation has been submitted earlier or concurrently for the same or any other degree.

Date: $2015 / 12(0.20$

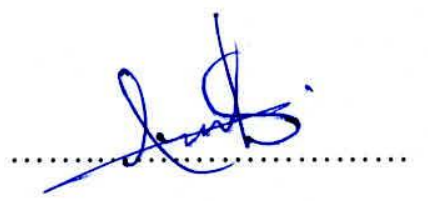

Signature of the Candidate

R. G .A Kumara

Faculty of Science

Postgraduate institute of Applied Science

Sri Jayawardanapura University

Nugegoda 
We certify that the declaration made by the candidate is true and this thesis is suitable for submission to the university for the purpose of evaluation

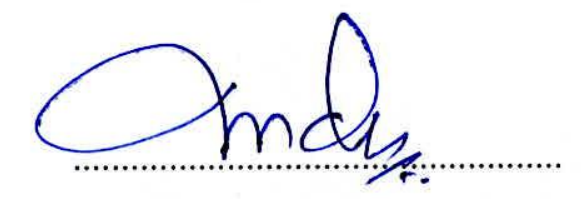

Prof. Arthur Bamunuarachchi

(Emeritus Prof.) University of Sri Jayawardanapura

Consultant Food Scientist \& Technologist

Date: -
Dr. (Mrs.) Indira Wickramasinghe

Senior Lecturer/ Department of Food Science and Technology

Faculty of Applied Sciences

University of Sri Jayewardenepura

Gangodawila

Nugegoda

Date:-..1.1............ 


\section{TABLE OF CONTENTS}

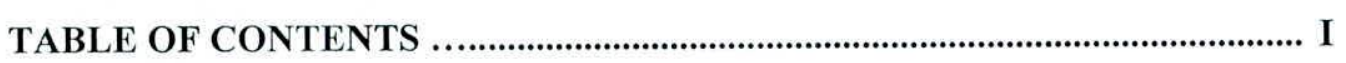

LIST OF FIGURES ……......................................................................

LIST OF TABLES ............................................................................. VI

ACKNOWLEDGEMENT .............................................................

ABSTRACT ....................................................................... VIII

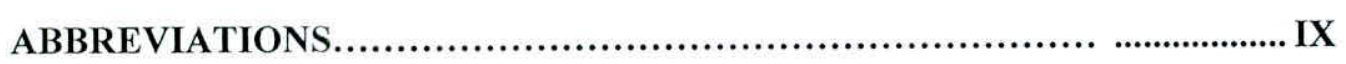

CHAPTER 1: INTRODUCTION.................................................. 01

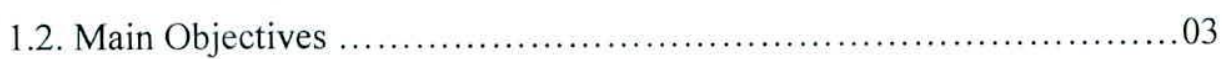

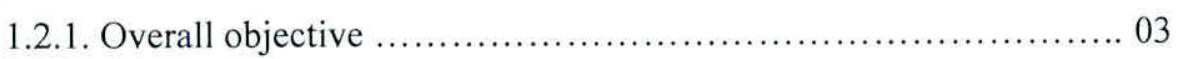

1.2.2. Specific objectives …........................................ 03

CHAPTER 2: LITRATURE REVIEWS ........................................... 04

2.1 Literature survey …................................................ 04

2.2. Chemistry and composition of the plant.................................. 07

2.3 The role of active constituents present in Centella asiatica ................. 11

2.3.1. CA Extracts \& wound healing ...................................... 14

2.3.2. Strengthening the weakened veins (Venous insufficiency).......... 15

2.3.3. Sedative and anxiolytic properties .................................... 16

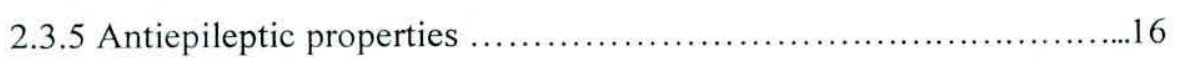

2.3.6. Cognitive and antioxidant properties ................................. 18

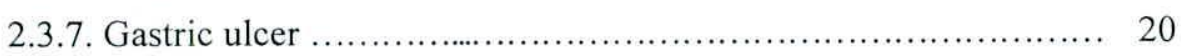

2.3.8. Antinociceptive and anti-inflammatory properties ..................21

2.4. Preliminary studies of bioassays for secondary metabolites ............. 23

2.4.1 Antioxidant activity ................................................... 23

2.4.1.1.Qualitative; TLC method .................................. 23

2.4.1..2 Quantitative; Spectrophotometry method ..........................24

2.4.2. Determination of total phenolic content .............................. 24

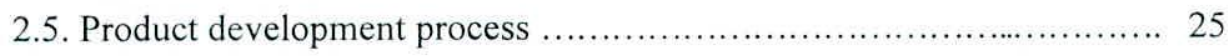

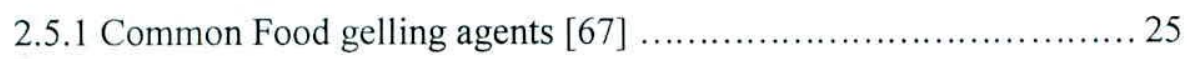




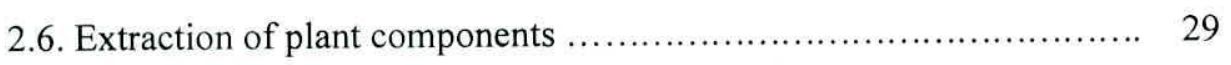

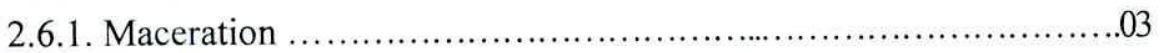

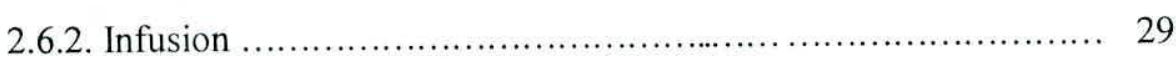

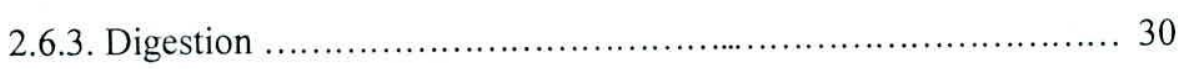

2.6.4. Decoction ........................................................ 30

2.7. Centella Asiatica Leaf Extract..................................... 32

2.7.1. Centella Asiatica Extract ................................... 33

CHAPTER 3 : MATERIALS AND METHODS ........................... 34

3.1. Purchasing and preparation of jellying agents ...................... 34

3.1.1. Materials ................................................. 34

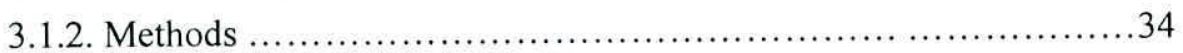

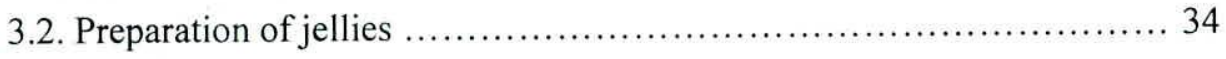

3.2.1 Materials ...................................................... 34

3.2.2. Method .................................................... 34

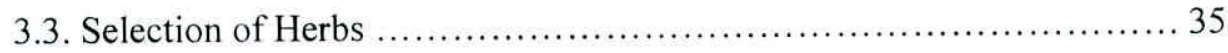

3.3.1. Materials ................................................... 35

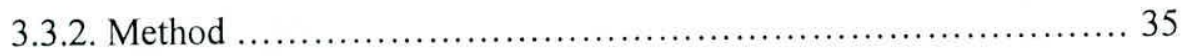

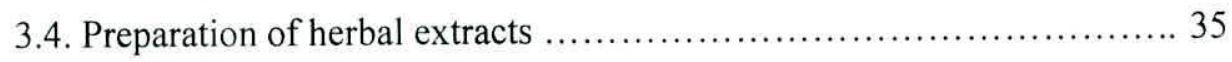

3.4.1. Materials ............................................. 35

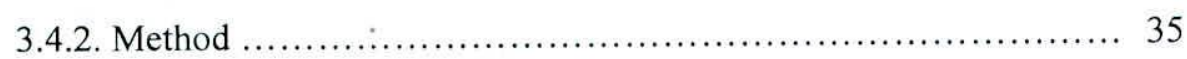

3.5. Preparation of products/Product development process ................ 36

3.5.1. Materials ..................................................... 36

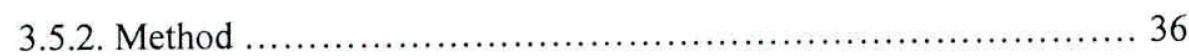

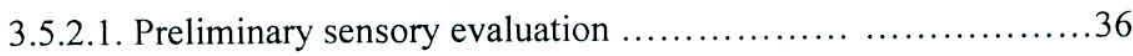

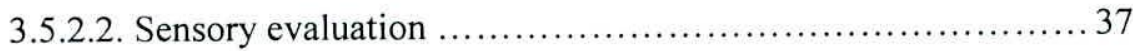

3.6. Phytochemical analysis of extracts/finished product .................... 37

3.6.1. Materials ....................................................... 37

3.6.2. Materials ................................................. 37

3.6.2.1 Methods to test for Saponins .......................... 37

3.6.2.2 Methods to test for Phenols ............................... 38 
3.6.2.3 Methods to test for Glycosides ........................ 38

3.7. Determination of antioxidant activity by DPPH assay .............. 38

3.7.1. Materials .............................................. 38

3.7.2. Methods ...................................................... 39

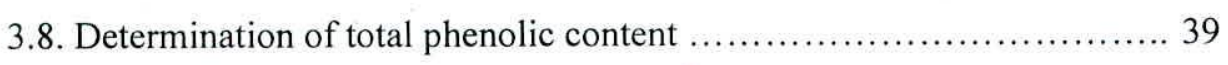

3.8.1. Materials ................................................. 39

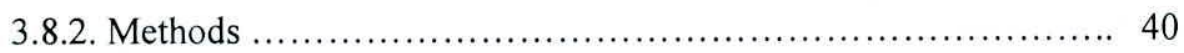

3.9.Determination of Nutrient content of the product .................. 41

3.9.1. Determination Moisture Content of Gotukola based jelly product.. 41

3.9.1.1. Materials. ............................................. 41

3.9.1.2. Methods............................................. 41

3.9.2. Determination of Protein content of Jelly product ............... 42

3.9.2.1. Materials .............................................. 42

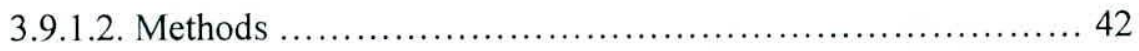

3.9.3. Determination of Fat content of Gotukola Based jelly product ...... 43

3.9.3.1. Materials ............................................ 43

3.9.3.2. Methods ............................................ 44

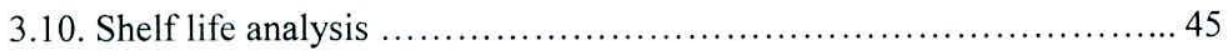

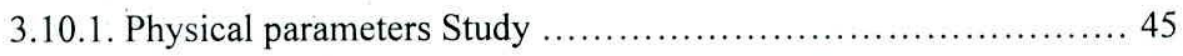

3.10.3.1. Materials ............................................ 45

3.10.2. Microbial Analysis ........................................ 45

3.10.2.1. Aerobic plate count............................... 45

3.10.2.1.1 Materials ....................................... 45

3.10.2.1.2. Methods ...................................... 45

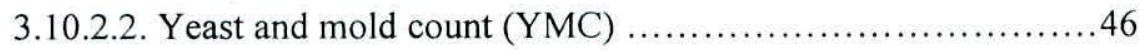

3.10.2.2.1 Materials ............................................ 46

3.10.2.2.2. Methods ......................................... 46

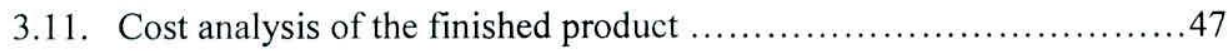

3.11.1. Methods ............................................. 47 


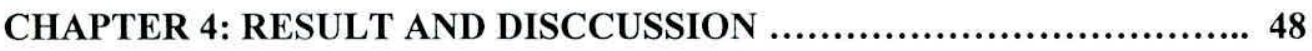

4.1. Purchasing and preparation of jellying agents ....................... 48

4.2. Development of the jelly product using deferent strength $(\mathrm{m} / \mathrm{v} \%)$......52

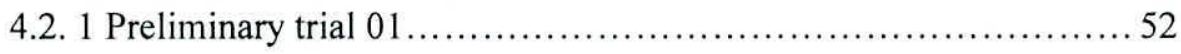

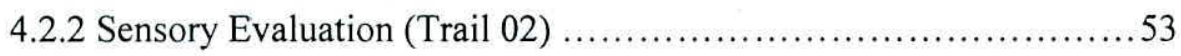

4.3. Phytochemical analysis of extracts/finished product ................... 57

4.4 Antioxidant activity bioassay (using Spectrophotometry method) ....... 59

4.5 Determination of total phenolic content .............................. 62

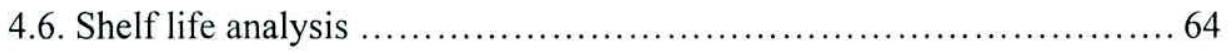

4.7 Proximate Results .................................................. 65

4.8. Cost analysis of the finished product .............................66

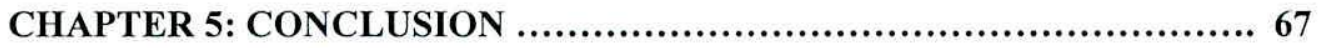

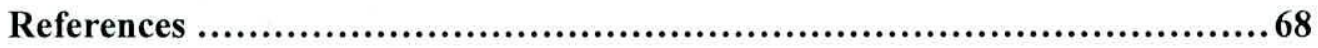

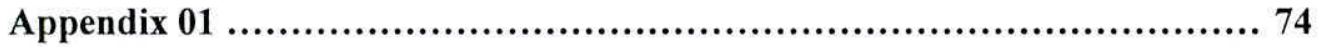

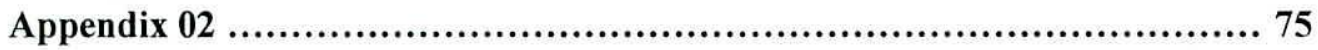

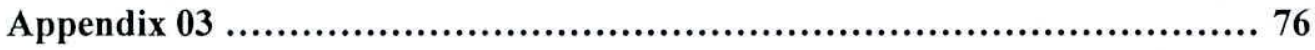

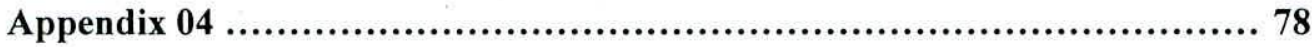




\section{LIST OF FIGURES}

Figure 2.1: Centella asiatica (Gotukola) Plant................................... 06

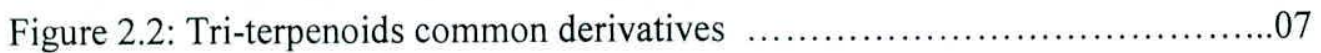

Figure 2.3: Major compounds present in Centilla Asiatica ....................... 10

Figure 2.4 Reduction of DPPH with presence of an antioxidant ................. 23

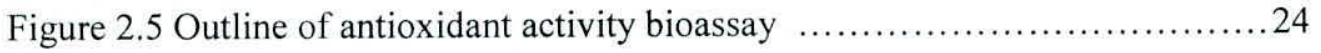

Figure 3.1: Rotary code system (Testing of Saponings) ......................... 38

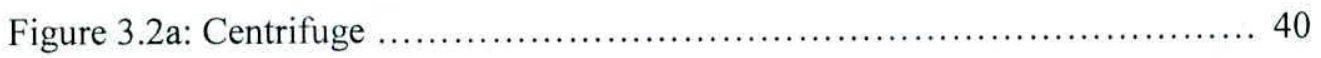

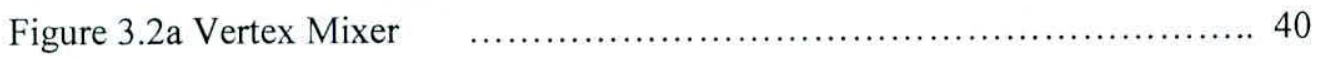

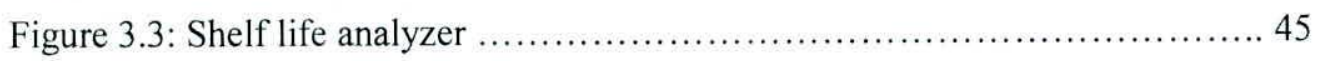

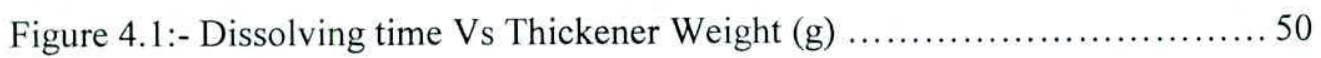

Figure 4.2: Physical State of China moss (A) and Carrageenan (B) ............. 50

Figure 4.3:- The Settling Time vs Thickener Weight ........................ 51

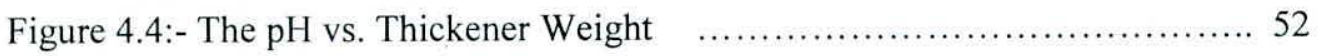

Figure 4.5:- Results for Triterpenoids (A) and Saponings (B) $\ldots \ldots \ldots \ldots \ldots \ldots \ldots$

Figure 4.6: Results for Phenols (B) and Glycosides (A) ...................... 58

Figure 4.7:- Reduction of DPPH in the presence of an antioxidant ............. 60

Figure 4.8: Plant extracts concentration series ................................ 60

Figure 4.9:- Plant extract concentration Vs $\%$ of Scavenging activity ..............6 61

Figure 4.10:- Product concentration Vs \%of Scavenging activity ................. 62

Figure 4.11:- BHT concentration Vs \%of Scavenging activity ................... 62

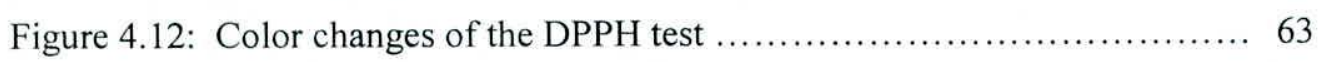

Figure 4.13:- Calibration curve of Gallic acid.............................. 64 


\section{LIST OF TABLE}

Table 2.1: The tri- terpene compounds of Centella asiatica......................... 08

Table 2.2: Concentration of gelling agents to form gels .......................... 26

Table: 3.1 . Tested parameters vs combination used (Trail 01) .................. 28

Table: 3.2 . Tested parameters vs combination used (Trail 02)..................40

Table 3.3: Material/ Apparatus and Reagents to measure Moisture content.......... 40

Table 3.4: Material/ Apparatus and Reagents to measure Protein Content.......... 45

Table 3.5: Material/ Apparatus and Reagents to measure Fat content ............ 56

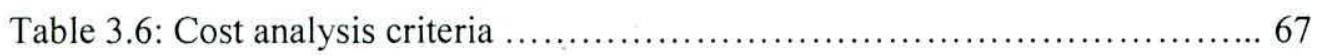

Table 4.1: Characters of gelling agents for the tested parameters ................ 49

Table 4.2:- Characters of the thickening agents............................. 53

Table 4.3:-Percentage combination of plant extract incorporated to make jelly.... 54

Table 4.4:- Mean scores for treatments with respect to sensory attributes............ 55

Table 4.5:- $\mathrm{P}$ values for treatments with different sensory attributes.............. 56

Table 4.6:- Phytochemical results....................................... 58

Table 4.7: Concentration Vs. $\%$ of scavenging activity.......................6 61

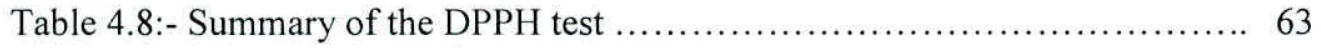

Table 4.9: Microbial results with shelf life .................................. 64

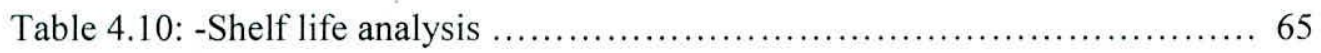

Table 4.11: Costing Details of the finished product .......................... 67 


\section{ACKNOWLEDGEMENT}

First and foremost I wish to express my deepest appreciation and sincere gratitude to my supervisor, Professor Artur Bamunuarachchi Research Professor, Sri Jayawardanapura University and Dr Indira Wickramasinghe, Sri jayawardanapura university, for your great insights, perspectives, guidance and sense of humor. Your excellence guidance, endless support, professional experience, advice and patience during my research work are highly acknowledged.

My sincere thanks go to the officers in the Postgraduate unit of Food MSc, especially Dr. Rupika perera Sri Jayawardanapura University Sri Lanka for helping in various ways to clarify the things related to my academic work in time with excellent cooperation and guidance. Sincere gratitude is also extended to the people who serve in the Department of Food Science.

Raigam Marketing Servies (Pvt.) Ltd. is gratefully appreciated for providing laboratory facilities to carry out this research. In R \& D Division and the Microbiological division of Raigam Marketing Servies (Pvt.) Ltd deserve a warm thank you, especially to Deputy General Manager Mr Nalin Gangodawila, who gives endless support to every aspect to perfect my study.

My warmest thanks belong to my Parents, Sister and Brother for their confidence in me and for being always so supportive and interested in my work.

Lastly, I should thank many individuals, friends and colleagues who have not been mentioned here personally in making this educational process a success. May be I could not have made it without your supports. 


\begin{abstract}
Functional foods are a novel and potential source of modern Food, Medicine, and Pharmaceutical industry. As the structurally diverse biologically active compounds possessing rich source of the traditional plants gained increased attention in the last decade as functional sources.

This study reports the isolation of plant extract of Gotu Kola, from the traditional way adapted in Aurveda as well as incorporation of Plant Extract to a jelly medium that can be used as a food to benefits to consumers as stated compressively in the Literature reviews. The nutritional aspects of them, selection of jelly based medium is done with the economic scale so that process cost and organolepticaly acceptance is tested. Carrageenan was the best material to use as a thickening agent for this study considering several factors like unit prices, Bulk densities, Easiness of use, settling time. It was noted that incorporated of $60 \%$ plant extract to product had the best results and it was kept under different conditions and noticed little variations of aroma only with the 03 months of shelf life study.

The antioxidant potential of the plant extract is tenfold higher than the product. The gel base used to develop the product does not show any DPPH potential. The antioxidant potential of the product is due to the herbal extract. The amount of total phenolic content is ranged from $500-800 \mathrm{mg} / \mathrm{GAE} / \mathrm{g}$ in all 03 stages of the product (Plant Extract, Finished Product, Gel base). Phytochemical tests represented the presence of triterpenoids, Saponin, Phenol, Glycosides, which functionally beneficial for human.
\end{abstract}

Keywords: Centlla asiatica (Gotukola), Jelly products, Centilla asiatica Extracts, Antioxidant potential of plants, Total phenolic content 


\title{
ABBREVIATIONS
}

\author{
CA - Centella Asiatica \\ CAE - CA Extracts \\ CNS - Central Nerve System \\ CCK- Cholesystokinin \\ DPPH - 2, 2'-diphenyl-1-picrylhydrazyl \\ DNA - Deoxyribo Nucleic Acid \\ HQOL- Health Related Quality Of Life \\ PE - Plant Extracts \\ TLC - Thin Layer Chromatography
}




\section{CHAPTER 1}

\section{INTRODUCTION}

The primary role of diet is to provide sufficient nutrients to meet the nutritional requirements of an individual. There is now increasing scientific evidence to support the hypothesis that some foods and food components have beneficial physiological and psychological effects over and above the provision of the basic nutrients.

Today, nutrition science has moved on from the classical concepts of avoiding nutrient deficiencies and basic nutritional adequacy to the concept of "positive" or "optimal" nutrition. The research focus has shifted more to the identification of biologically active components in foods that have the potential to optimize physical and mental well-being and which may also reduce the risk of disease (EUFIC, 2015)

Many traditional food products including fruits, vegetables and whole grains have been found to contain components with potential health benefits. In addition to these foods, new foods are being developed to enhance or incorporate these beneficial components for their health benefits or desirable physiological effects

Jelly and gel is that generally jelly is a dessert made by boiling gelatin, sugar and some flavoring (often derived from fruit) and allowing it to set while gel is a semi-solid to almost solid colloid of a solid and a liquid, such as jelly, cheese or opal or gel.The traditional uses of seaweed as food and, to a lesser extent, as animal feed and fertilizer supplements remain important, but in most parts of the world it is as raw materials for certain chemical products that marine algae are now chiefly valued. The especial role of seaweeds as food in 
the Far East and the prospects for a wider contribution by seaweeds to human nutrition (FAO,1973)

Centella asiatica, also known as Gotu Kola in Sinhala, is an annual herb native to India, Australia and Asia. Its leaves are eaten as a vegetable and it is also an important herb in the traditional medicine systems of those places. Research has revealed support for several of Centella asiatica's purported health benefits. Centella asiatica is traditionally used as a medicinal herbs and alternative medicine in treating numerous kinds of diseases comprehensively describe in literature.

The use of Centellain food and beverages has increased over the years. Its potential antioxidant and neuroprotective activity has been widely claimed in many reports and basically is very much related to its properties and mechanism of action of the plant's bioactive constituents namely the asiaticoside, asiatic acid, madecassoside and madecassic acid

Centellais commonly eaten fresh as vegetable especially among the locals the salads are eatentogether with the main meal and can act as an appetizer. Beside eaten raw, it can be cooked as a part of a soupor as a main vegetables. Due to its mild bitterness it is always cook and served with the addition of coconut milk or shredded coconut and sometimes sweet potatoes and potatoes are added.

Since the Centella is very popular as a vegetable, it is available everywhere in the markets and supermarkets as a vegetable and therapeutic use, the whole plant including leaves; stem and root is consumed (Brinkhauset al., 2000). It is used as health tonic and processed into cordial drinks and ready to drink juice (Mohdllham, 1998). The fresh plants are also blended to make drink and juice. Centella herbal noodles have been developed. The best 
formulation for the noodle is found to be $20 \%$ Centella extract, $5 \mathrm{~g}$ of salt with noodles base. This formulation has provided the best sensory acceptance and highest presence of flavonoids (cathechin, quercetin and rutin).

Natural plant-based extracts can conserve the appearance, taste and quality of food products with minimal impact on their flavor, color and odor profiles. With natural plant extracts customers benefit from proprietary flavor extracts developed with effective antioxidant properties for delaying oxidation and increasing shelf-life. These versatile, natural solutions are label friendly alternatives to traditional tocopherols or conventional synthetic antioxidants commonly used in the food industry.

\subsection{Main Objectives}

\section{Overall objective}

The aim of this research was to develop a ready to eat (RTE) product using of Centilla Asiatica plant extract with organoleptic acceptance.

\section{Specific objectives}

$\checkmark$ To develop an economically beneficial jelly base

$\checkmark$ To extraction of plant decoction from Centilla Asiatica

$\checkmark$ To develop the jelly product using deferent strength ( $\mathrm{m} / \mathrm{v} \%)$ of the herbal extracts

$\checkmark$ To conduct Phytochemical analysis of extracts/finished jelly products

$\checkmark$ To determine the Antioxidant activity and the Total Phenolic Content.

$\checkmark$ To determine the proximate analysis (Selective) of the product

$\checkmark$ To study for the Shelf life \& Cost analysis of the finished product 